\title{
Nanoencapsulation of Rose-Hip Oil Prevents Oil Oxidation and Allows Obtainment of Gel and Film Topical Formulations
}

\author{
Renata V. Contri, ${ }^{1,4}$ Irene C. Kulkamp-Guerreiro, ${ }^{1,2}$ Sheila Janine da Silva, ${ }^{2}$ Luiza A. Frank, ${ }^{1}$ \\ Adriana R. Pohlmann, ${ }^{1,3}$ and Silvia S. Guterres ${ }^{1,2}$
}

Received 15 April 2015; accepted 24 July 2015; published online 17 September 2015

\begin{abstract}
The rose-hip oil holds skin regenerating properties with applications in the dermatological and cosmetic area. Its nanoencapsulation might favor the oil stability and its incorporation into hydrophilic formulations, besides increasing the contact with the skin and prolonging its effect. The aim of the present investigation was to develop suitable rose-hip-oil-loaded nanocapsules, to verify the nanocapsule effect on the UV-induced oxidation of the oil and to obtain topical formulations by the incorporation of the nanocapsules into chitosan gel and film. The rose-hip oil $(500$ or $600 \mu \mathrm{L})$, polymer (Eudragit RS100®, 100 or $200 \mathrm{mg}$ ), and acetone ( 50 or $100 \mathrm{~mL}$ ) contents were separately varied aiming to obtain an adequate size distribution. The results led to a combination of the factors acetone and oil. The developed formulation showed average diameter of $158 \pm 6 \mathrm{~nm}$ with low polydispersity, $\mathrm{pH}$ of $5.8 \pm 0.9$, zeta potential of +9.8 $\pm 1.5 \mathrm{mV}$, rose-hip oil content of $54 \pm 1 \mu \mathrm{L} / \mathrm{mL}$ and tendency to reversible creaming. No differences were observed in the nanocapsules properties after storage. The nanoencapsulation of rose-hip oil decreased the UVA and UVC oxidation of the oil. The chitosan gel and film containing rose-hip-oil-loaded nanocapsules showed suitable properties for cutaneous use. In conclusion, it was possible to successfully obtain rose-hip-oil-loaded nanocapsules and to confirm the nanocapsules effect in protecting the oil from the UV rays. The chitosan gel and film were considered interesting alternatives for incorporating the nanoencapsulated rose-hip oil, combining the advantages of the nanoparticles to the advantages of chitosan.
\end{abstract}

KEY WORDS: chitosan film; chitosan gel; polymeric nanocapsules; rose-hip oil; UV-induced oxidation.

\section{INTRODUCTION}

The rose-hip or rosa mosqueta oil is mainly obtained from the seeds of the plant Rosa rubiginosa found in Central Europe and the Andean region (1). This oil, presenting a greasy non-volatile aspect, is widely composed of unsaturated fat acids like the linoleic, oleic, and linolenic acids, and also of poliphenols, carotenoids, and transretinoic acid, representing therefore an alternative for accelerating the skin regenerating process (1-3). Pigmentation, ulceration, and scaring problems can be treated with rose-hip oil (4). Its use includes dermatological and cosmetic applications, used in its plain form or in formulations at concentrations ranging from 2 to $30 \%$.

Nanoparticles for dermatological and cosmetic use have been extensively studied, in the past years (5-7). The

\footnotetext{
${ }^{1}$ Programa de Pós-Graduação em Ciências Farmacêuticas, Universidade Federal do Rio Grande do Sul, Av. Ipiranga, 2752/405, CEP 90610-000, Porto Alegre, RS, Brazil.

${ }^{2}$ Faculdade de Farmácia, Universidade Federal do Rio Grande do Sul, Porto Alegre, Brazil.

${ }^{3}$ Instituto de Química, Universidade Federal do Rio Grande do Sul, Porto Alegre, Brazil.

${ }^{4}$ To whom correspondence should be addressed. (e-mail: renata.contri@gmail.com)
}

nanoencapsulation advantages include a more strict contact with the stratum corneum, due to a great skin adhesion of the nanostructures (8) and a controlled release of the active substances (9). Those advantages lead to a more prolonged action $(10,11)$ and increase in efficacy $(12)$. Due to the size of around $300 \mathrm{~nm}$, those nanoparticles used on the skin are not able to cross the intact stratum corneum (7). Benefits regarding the formulating process and stability achievement should also be taken into consideration. Oil-loaded nanocapsules in aqueous suspension represent an easy way to incorporate oils of interest in cosmetic formulations, especially formulations containing low lipid content, such as gels. Although it was previously proven that the nanoencapsulation protects active substances from physical or chemical degradation (13-15), the protection of the nanocapsules to the degradation of the oily core has not been evaluated. This is of even more interest when the oil core is used by its pharmacological or cosmetic properties, which is the case of rose-hip oil.

The nanoencapsulation of rose-hip oil, among other vegetable oils, has been previously proposed by our research group using Eudragit RS100® as polymer (16). The polymer was chosen due to its biocompatibility and cationic properties, which increase skin adhesion (8). However, such nanocapsule suspension presented some aspects to be improved: particles in the micrometer range, a large size distribution, and high 
creaming of the nanocapsules. Nevertheless, the viscosity of formulations were observed to be very low, similar to the viscosity of pure water (16), not favoring the topical application. In this context, the obtainment of final formulations is considered essential; therefore, the selection of an adequate semisolid vehicle is a crucial step.

Chitosan, a hydrogel-forming and film-forming polymer (17), has been proposed as gel vehicle for polymeric nanocapsules (8), liposomes (18), niossomes (19), and solid lipid nanoparticles (20), as well as film vehicle for polymeric nanoparticles (21) and silver nanoparticles (22). Some interesting properties of this polymer such as bioadhesion, biocompatibility, and wound healing properties (23-25) turn chitosan formulations into great candidates for the topical application of rose-hip-oil-loaded nanocapsules.

Thus, the objective of the present article was to improve the nanoencapsulation of rose-hip oil in terms of homogeneity of nanocapsule sizes avoiding the presence of particles with more than $1000 \mathrm{~nm}$, by varying the component contents. Also, the effect of the nanocapsules on the UVA- and UVC-induced oxidation of the rose-hip oil and the development of final topical gel and film formulations, using the biopolymer chitosan, was investigated.

\section{MATERIALS AND METHODS}

\section{Materials}

The rose-hip oil was purchased from Embacaps (Porto Alegre, Brazil). Eudragit RS 100® (approximate molecular weight of $32,000 \mathrm{~g} / \mathrm{mol}$ ) and polysorbate 80 were purchased from Degussa (Darmstadt, Germany) and Labsynth (São Paulo, Brazil), respectively. Chitosan (medium molecular weight and deacetylation degree of $77 \%$ ) and tripolyphosphate were obtained from Sigma-Aldrich (São Paulo, Brazil). Lactic acid at a concentration of $85 \%$, glycerin, decamethylcyclopentasiloxane or volatile silicone fluid, and sodium pyrrolidone carboxylic acid (Na-PCA) were obtained from Via Farma (São Paulo, Brazil). Acetone and ethyl acetate of analytical grade were obtained from Vetec (Rio de Janeiro, Brazil), while acetonitrile of HPLC grade was obtained from Tedia (Sao Paulo, Brazil).

\section{Nanocapsule Production and Formulation Improvement}

The nanocapsule aqueous suspensions were obtained by means of the interfacial deposition of pre-formed polymer method in which an organic phase containing the organic solvent (acetone), the rose-hip oil and the nanocapsule shellforming-polymer (Eudragit RS100®), was injected into an aqueous phase containing polysorbate 80 as hydrophilic surfactant. The acetone and part of the water (until volume of $10 \mathrm{~mL}$ ) were then eliminated at a rotary evaporator (R-114, Büchi).

Based on the previous formulation of nanoencapsulated rose-hip oil, obtained with slight modifications from CONTRI and co-workers (16) ( $\left.\mathrm{NC}_{\text {Original }}\right)$, three components, the rosehip oil (NC-A), the polymer (NC-B), and the acetone (NC-C), were varied in order to attempt an improvement of the formulation regarding the percentage of microparticles in the formulation and the homogeneity of the system, observing the average nanoparticle diameter, as well (Table I). Such data [percentage of particles above $1000 \mathrm{~nm}, \mathrm{D}(4,3)$, and SPAN value] were obtained by means of laser diffraction analyses after dilution in ultrapure water (Mastersizer, Malvern), using the refraction index of Eudragit RS100® (1.38). Based on the results, we developed a more suitable formulation $\left(\mathrm{NC}_{\text {Improved }}\right)$ which was further analyzed as described below.

The polydispersity values (SPAN values) were determined as follows (Eq. 1):

$$
S P A N=\frac{\left(D_{0.9}-D_{0.1}\right)}{D_{0.5}}
$$

where $D_{0.1}, D_{0.5}$, and $D_{0.9}$ are the diameters that correspond to 10,50 , and $90 \%$, respectively, in the size distribution.

\section{Nanocapsule Characterization}

The improved nanocapsule $\left(\mathrm{NC}_{\text {Improved }}\right)$ formulation was further characterized in terms of average diameter, polydispersity, zeta potential, nanocapsule concentration, oil content, and pre-stability. The diameter and polidispersity were evaluated by light scattering (Zetasizer, Malvern) after dilution in ultrapure water $(1: 500 v / v)$, using the refraction index of 1.38. To guarantee that the oil was encapsulated within the nanocapsules, three consecutive measurements of the size distribution profiles were analyzed. Dynamic systems present different profiles among the measurements, which would indicate formation of nanocapsules and nanoemulsion simultaneously, while stable formulations present similar profiles among the consecutive measurements (26). The zeta potential was evaluated by eletroforetic mobility (Zetasizer, Malvern) after dilution in $10 \mathrm{mM} \mathrm{NaCl}$ solution $(1: 500 v / v)$. The $\mathrm{pH}$ was analyzed by potentiometry (Micronal) by direct measurement in the suspensions. The number of particles per milliliter (concentration) was determined by turbidimetry at $395 \mathrm{~nm}$ (spectrophotometer Femto 600 Plus) by several dilutions in ultrapure water. The determinantion of the oil content was performed by a matrix superposition method at $269 \mathrm{~nm}$ (spectrophotometer Femto 600 Plus) by dilutions in ethyl acetate/acetonitrile. The morphology of the nanocapsules was evaluated by transmission electron microscopy (TEM; JEM 1200 Exll operating at $80 \mathrm{~K}$ ). The aqueous suspension sample was deposited on a Formvar/Carbon grid and negatively stained with uranyl acetate solution $(2 \% w / v)$.

The pre-stability of the aqueous nanocapsule suspension was analyzed by its resistance to centrifugation (MTDIIIPLUS, $30 \mathrm{~min}$ under $1210 \times g$ ), by its tendency to physical instability phenomena using the multiple light scattering technique (Turbiscan LAB, Formulaction, at $25^{\circ} \mathrm{C}$ for $12 \mathrm{~h}$ ), and by the evaluation of the formulation properties under storage at room temperature and at $40^{\circ} \mathrm{C}$. After 30 days, in the mentioned conditions, the formulation was again characterized in terms of average diameter, polydispersity, zeta potential, and $\mathrm{pH}$, as described above.

\section{In Vitro Oxidation Study}

The effect of the nanoencapsulation on the rose-hip oil oxidation process was evaluated $(n=3)$, considering the possibility of increasing the formulation stability under UV radiation. The rose-hip oil oxidation under UVA and UVC lights 
Table I. Composition of Nanocapsule Colloidal Dispersion $(10 \mathrm{~mL})$

\begin{tabular}{|c|c|c|c|c|c|}
\hline Formulation & Acetone $(\mathrm{mL})$ & Polymer (Eudragit RS 100®) (mg) & Rose-hip oil $(\mu \mathrm{L})$ & Ultrapure water $(\mathrm{mL})$ & Polysorbate $80(\mathrm{mg})$ \\
\hline $\mathrm{NC}_{\text {Original }}$ & 54 & 200 & 660 & 106 & 152 \\
\hline NC-A & 54 & 200 & $\underline{500}$ & 106 & 152 \\
\hline NC-B & 54 & $\underline{300}$ & $\overline{660}$ & 106 & 152 \\
\hline $\mathrm{NC}-\mathrm{C}$ & $\underline{100}$ & $\overline{200}$ & 660 & 106 & 152 \\
\hline $\mathrm{NC}_{\text {Improved }}$ & $\overline{100}$ & 200 & $\underline{500}$ & 106 & 152 \\
\hline
\end{tabular}

Underlined numbers indicate the changes performed in relation to the prior formulation $\left(\mathrm{NC}_{\text {Original }}\right)$

was studied by means of the lipoperoxidation process, quantifying the fluorescent product obtained by the reaction of malondialdehyde and thiobarbituric acid at $532 \mathrm{~nm}$ by UV spectroscopy (27). The malondialdehyde is one of the most frequently used indicators of lipid peroxidation.

The nanocapsule aqueous suspensions $\left(\mathrm{NC}_{\text {Improved }}\right)$ were placed under UVA and UVC light sources for $24 \mathrm{~h}$. At predetermined time points, aliquots from the formulations were withdrawn and mixed with trichloreacetic acid $(12 \% \mathrm{w} / \mathrm{v}$ in water) and thiobarbituric acid $(0.73 \% w / v$ in water $)$. The fluorescent color appeared when the formulations were placed in a water bath at $100^{\circ} \mathrm{C}$ for $30 \mathrm{~min}$. The mixture obtained was centrifuged for $12 \mathrm{~min}$ at $10,000 \mathrm{rpm}$ and $25^{\circ} \mathrm{C}$. The supernatant was obtained and analyzed regarding its absorbance at $532 \mathrm{~nm}$. The nanocapsule aqueous suspension was compared to a dispersion of the oil in water and polysorbate 80 , at the same concentrations of rose-hip oil and surfactant. The values obtained were discounted from the values obtained at time zero (treating the formulations in similar conditions in except for the period under UV radiation). It is important to notice that, previously to the quantification, growing fluorescence curves were obtained by adding different concentrations of malondialdehyde aqueous solution (obtained by a standard solution) at $100^{\circ} \mathrm{C}$ and centrifugating afterward. The linearity was obtained in the range from 0.0036 to $0.022 \mathrm{mM}$ of malondialdehyde.

\section{Development and Characterization of Final Dermal Formulations Based on Chitosan}

Based on the fact that the aqueous nanocapsule suspensions developed are difficult to apply on the skin, besides the possibility of instability and microbial contamination, two final formulations based on chitosan (gel and film) were proposed for the cosmetic and dermatological use of rose-hip oil (Table II). The preparation procedure is described below.

Gel

The chitosan gel containing rose-hip oil nanocapsules was obtained by simple mixing of chitosan, nanocapsule aqueous suspension, lactic acid, and sodium pyrrolidone carboxylic acid. Then, volatile silicone and diazolidinyl urea were added to the formulation (28). The innovative hydrogel was obtained in triplicate of batches. The characterization of the gel formulation was performed regarding size distribution, viscosity, $\mathrm{pH}$, and pre-stability. The size distribution analysis was evaluated by laser diffraction (Masterizer, Malvern) performing a dilution in ultrapure water. The viscosity was assessed by rotary viscosimetry (Brookfield® LV-DV-II+Pro, spindle SC4-25 at $25^{\circ} \mathrm{C}$ ). The $\mathrm{pH}$ value was evaluated by potentiometry (Micronal) after dilution in ultrapure water $(1: 10 w / v)$. The tendency to instability phenomena was analyzed by multiple light scattering (Turbiscan LAB, Formulaction) by direct measurement at $25^{\circ} \mathrm{C}$ for $12 \mathrm{~h}$.

Film

The chitosan film containing rose-hip oil nanocapsules was obtained by mixing of chitosan, nanocapsule aqueous suspension, lactic acid, and glycerin, followed by solvent casting (17). The water was eliminated by keeping $1 \mathrm{~g}$ of the formulation in recipients of $3 \mathrm{~cm}$ of diameter under desiccator for 15 days. After drying, tripolyphosphate aqueous solution was added to each recipient, increasing the rigidity of the films and allowing the removal from the recipients. The innovative film containing nanocapsules was obtained in triplicate of batches. The characterization of the film was performed regarding weight, thickness, $\mathrm{pH}$, presence of nanocapsules, and water absorption capacity. The thickness was assessed by direct measurements of the films formed using a dial thickness gauge (No. 7301®, Mitutoyo, Japan). The $\mathrm{pH}$ and presence of nanocapsules were determined, after disruption of the films with ultrapure water and vortex mixing, by potentiometry (Micronal) and by laser diffraction (Masterizer, Malvern), respectively. The water absorption was determined by keeping films immersed in ultrapure water $\left(37^{\circ} \mathrm{C}\right)$. Then, at predetermined times, the film weights were determined.

\section{Statistical Analyses}

The statistical analysis of the characterization properties were performed by ANOVA, followed by Dunnet's test, for multiple groups and Student's $t$ test for two groups. In both cases, differences were considered significant for $\alpha=0.05$.

Table II. Composition of Dermal Chitosan Formulations Containing Rose-Hip-Oil-Loaded Nanocapsules

\begin{tabular}{lll}
\hline Components & Gel & Film \\
\hline Chitosan & $2.5 \%$ & $1 \%$ \\
Lactic acid & $1 \%$ & $1 \%$ \\
Carboxylic pirrolidon acid sodium salt & $2 \%$ & - \\
Decamethylcyclopentasiloxane & $3 \%$ & - \\
Diazolidinyl urea & $0.5 \%$ & - \\
Glycerin & - & $10 \%$ \\
Tripolyphosphate & - & $2 \%$ \\
Rose-hip oil (nanoencapsulated) & $5 \%$ & $5 \%$ \\
\hline
\end{tabular}




\section{RESULTS AND DISCUSSION}

\section{Nanocapsule Improvement}

The changes in the previous developed formulation ( $\mathrm{NC}_{\text {Original }}$ ) (16) were performed in order to obtain higher homogeneity in terms of nanoparticle sizes, also aiming to exclude the simultaneous presence of particles of more than $1000 \mathrm{~nm}$. Therefore, the contents of three components of the organic phase were varied, compared to the prior formulation. Changing the amount of rose-hip oil (NC-A), polymer (NC$\mathrm{B})$, and acetone (NC-C), three results were evaluated: the SPAN value, the percentage of microparticles, and the average diameter. The values observed for the triplicate of batches are shown in Fig. 1.

When the content of acetone was increased (NC-C), the SPAN value is significantly decreased (Fig. 1a). On the other side, decreasing the amount of oil (NC-A), the SPAN value is significantly decreased. Similar results are obtained for the percentage of microparticles (Fig. 1b) and average diameter (Fig. 1c). The SPAN value reduced 1.5 and 1.9 times by decreasing the oil and increasing the acetone, respectively. The microparticle percentage reduced 2 times and 11.8 times by decreasing the oil and increasing the acetone, respectively. The average diameter reduced 1.4 and 1.8 times by decreasing the oil and increasing the acetone, respectively. Those findings indicate that smaller oil droplets are obtained in the organic phase by reducing the oil and increasing the acetone. Such smaller droplets in the organic phase lead to smaller average diameters, improving the homogeneity of the system. The encapsulation efficiency is probably affected by increasing the acetone in the organic phase, since, as previously mentioned, smaller oil droplets are formed during particle production. The increase in the amount of acetone is not considered a production problem because the acetone is completely evaporated during the process and could be reused. Also, it is important to notice that the increase in particle sizes due to increase of oil content suggests that the rose-hip oil is encapsulated within the nanocapsules. The polymer content (NC-B) did not show influence on the three results analyzed. The excess of the polymer in relation to the original formulation was probably not able to improve the formulation since residual polymer was observed after evaporating the solvents under reduced pressure, for the formulations with $300 \mathrm{mg}$ of Eudragit RS $100 \AA$. Therefore, the original amount of polymer was considered ideal, since it was able to be successfully placed around the oil droplets.

From the results obtained after the component changes, it was possible to conclude that another formulation, called $\mathrm{NC}_{\text {Improved, }}$ combining the changes performed in the oil and in the acetone content should be prepared. Increasing the amount of acetone (from 54 to $100 \mathrm{~mL}$ ) and simultaneously decreasing the amount of oil (from 660 to $500 \mu \mathrm{L}$ ) led to smaller particles sizes (from $333 \pm 37$ to $162 \pm 10 \mathrm{~nm}$ ), lower SPAN values (from $3.2 \pm 0.7$ to $1.5 \pm 0.1$ ), and lower percentage of microparticle (from $4.7 \pm 0.8$ to $0.2 \pm 0.3 \%$ ), improving the formulation. The average diameter and the SPAN value were reduced 2.1 times while the percentage of microparticles was reduced in 23.5 times, respectively after formulation improvement.
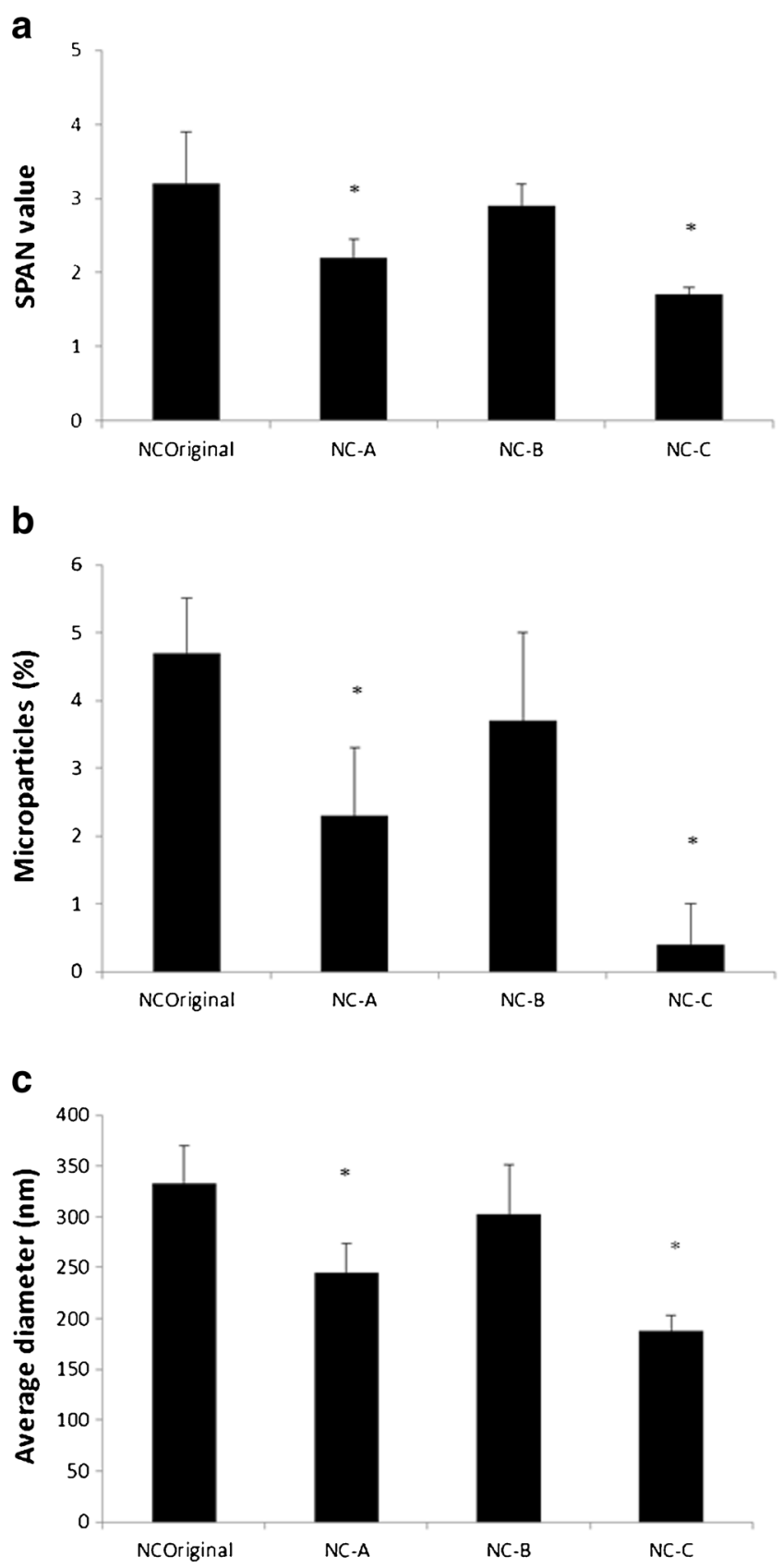

Fig. 1. SPAN values (a), percentage of microparticles (b), and average diameter (c) of nanocapsule colloidal dispersion. *Statistical difference compared to $\mathrm{NC}_{\text {Original }}(p<0.05)$

\section{Nanoparticle Properties}

The improved nanocapsule aqueous suspension was further characterized. The nanocapsule presented average diameter of $158 \pm 6 \mathrm{~nm}$ by means of light scattering analyses, similar to the value found by laser diffraction (Nanocapsules improvement). When comparing the three consecutive measurements of the size distribution profiles of the improved formulation, very similar profiles were observed indicating stable systems regarding the particle size. Therefore, it is concluded that the oil is encapsulated within the nanocapsules. The polydispersity index obtained by the mentioned 


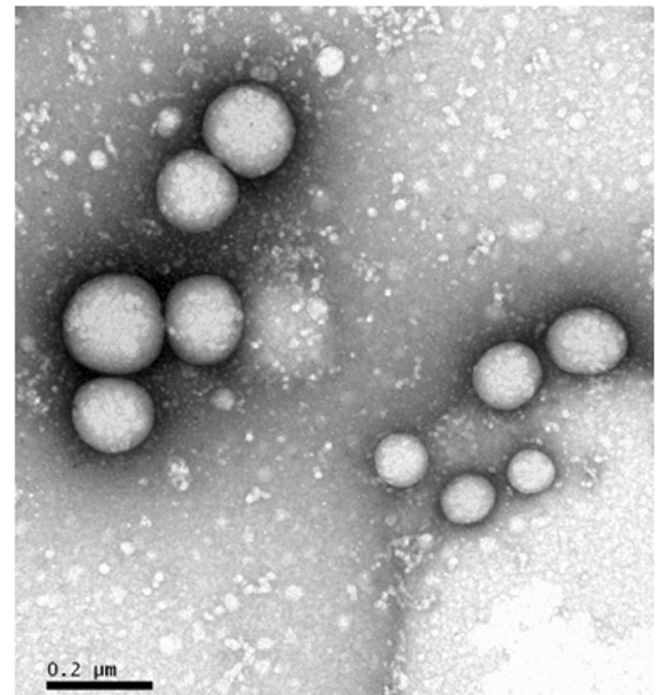

Fig. 2. Transmission electron microscopy image of rose-hip-oil-loaded nanocapsules

technique was $0.12 \pm 0.02$, proving a great homogeneity in the system regarding the nanocapsule sizes. By means of electron microscopy, the nanocapsules were found to be spherical, with homogeneous size, and values close to that obtained by laser diffraction and light scattering (Fig. 2). The zeta potential was found to be $+9.8 \pm 1.5$ due to the cationic properties of Eudragit RS 100®, used as nanocapsule shell. The positive zeta potential can increase the contact of the nanocapsules with the skin, due to negative charge of the tissue, as previously observed for the vaginal mucosae (29). The value obtained for the $\mathrm{pH}$ was $5.8 \pm 0.9$, which is in agreement with previous similar nanocapsule aqueous suspensions (30). The nanocapsule aqueous suspension presented $5.37 \pm 0.46 \times 10^{13}$ particles per milliliter, a higher value than the ones previously obtained by encapsulating vegetable oils in Eudragit RS100® nanocapsules (16). This result was expected since the volumetric fraction was two times higher and the acetone and oil amounts were changed, leading to particles smaller in size and higher in number.

Regarding the rose-hip oil content in the formulation, a UV methodology was previously validated. The linearity was obtained by adding a constant amount of polymer and polysorbate 80 (the same amount as present when the oil amount in the formulation was determined) and adding different amounts of rose-hip oil from a standard solution $(100 \mu \mathrm{L} / \mathrm{mL})$ to the five different curve points ( 1 to $7 \mu \mathrm{L} / \mathrm{mL}$ ). The linearity of the method was confirmed (correlation coefficient of more than 0.99 ), and the method was considered precise and accurate. Using the referred validated methodology, the amount of rosehip oil in the formulation was found to be $54 \pm 1 \mu \mathrm{L} / \mathrm{mL}$ (108\% of the theoretical value). The percentage of rose-hip oil in the improved nanocapsule formulation was $5 \%$, which is in the range of usual topically applied formulations containing the oil. Therefore, by means of nanocapsule improvement, it was possible to successfully encapsulate a high amount of vegetable oil indicating the obtainment of an efficient skin regenerating formulation. Eudragit RS 100® nananocapsules present great potential for controlled delivery of lipophilic substances as previously proven with capsaicinoid-loaded nanocapsules (31), which suggests that the actives present in the rose-hip oil, such as transretinoic acid, will be delivered in a controlled way when applied on the skin.

The nanocapsule formulation was additionally characterized in terms of pre-stability. The formulation was analyzed by centrifugation and by multiple light scattering (predetermined time points for a total period of $12 \mathrm{~h}$ ), to investigate its tendency to physical instability phenomena, such as sedimentation, creaming, or particle agglomeration. No instability phenomena were observed after the centrifugation process, suggesting adequate oil nanoencapsulation. Regarding multiple light scattering analysis, Fig. 3 shows the variation of the backscattering signal relative to the first measurement ( $\triangle \mathrm{BS})$. The light backscattering is shown since the transmittance was null (data not shown). It is possible to observe an increase in the $\triangle \mathrm{BS}$ with the time, at the top of the cell. The opposite occurred at the bottom of the cell, where a decrease in $\Delta \mathrm{BS}$ is observed as a function of the time. This is a typical creaming profile, indicating the migration of particles to the top of the cell, as previously described (16).

In order to verify if the creaming was reversible for the nanocapsule suspension here described, the nanocapsule basic properties (average diameter, polydispersity, and zeta potential) were again measured after 30 days under storage at room temperature and at $40^{\circ} \mathrm{C}$. The formulations were slightly homogenized by hand and analyzed (Table III). No significant differences were observed in the majority of nanocapsule properties after storage, confirming that the creaming did not influence the average size, the homogeneity regarding

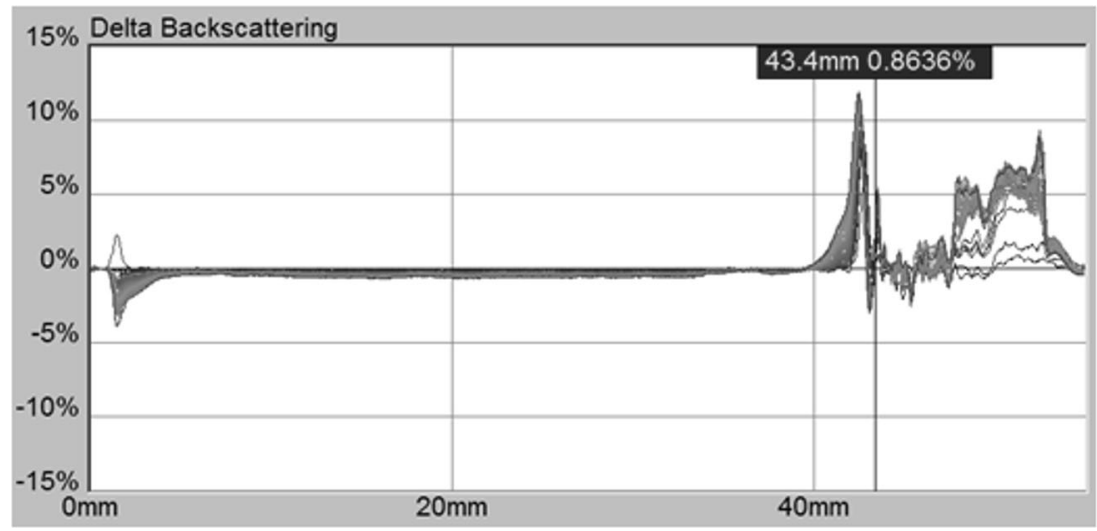

Fig. 3. Delta backscattering analysis of rose-hip-oil-loaded nanocapsule colloidal dispersion 
Table III. Properties of Rose-Hip-Oil-Loaded Nanocapsule Colloidal Dispersion After Storage at Room Temperature and at $40^{\circ} \mathrm{C}$

\begin{tabular}{|c|c|c|c|c|c|c|}
\hline Storage period/condition & Size (laser diffraction) & SPAN & Size (light scattering) & PDI & Zeta potential & $\mathrm{pH}$ \\
\hline 30 days/room temperature & $161 \pm 9$ & $1.4 \pm 0.1$ & $158 \pm 3$ & $0.13 \pm 0.01$ & $9.2 \pm 0.7$ & $5.4 \pm 0.3$ \\
\hline 30 days $/ 40^{\circ} \mathrm{C}$ & $146 \pm 7$ & $1.2 \pm 0.1$ & $155 \pm 2$ & $0.14 \pm 0.07$ & $10.2 \pm 0.8$ & $4.5 \pm 0.1 *$ \\
\hline
\end{tabular}

*Statistical difference compared to the value obtained right after production

particles sizes, or the surface charge, being considered a reversible physical instability phenomenon. The $\mathrm{pH}$ values, also measured after storage period, presented significant decay only when stored at $40^{\circ} \mathrm{C}$. Such decay was previously described for similar nanocapsules, and it is probably due to the partial hydrolysis of the polymer side groups not causing short-time destabilization system (16).

\section{Effect of the Nanocapsules in Protecting the Rose-Hip Oil from Oxidation due to $\mathrm{UV}$ Light}

The nanocapsule aqueous suspension and a dispersion of oil in the same amount of polysorbate 80 and water were put under UVA and UVC light for $24 \mathrm{~h}$. Aliquots were withdrawn and analyzed regarding the presence of malondialdehyde, after its reaction with tiobarbituric acid, forming a colored compound. The unsaturated fatty acids are probably the oxidized compounds. The malondialdehyde content $(\mathrm{mM})$ as a function of the time (Fig. 4) was determined by means of a pre-validated methodology. Considering the UVA light, there is a significant
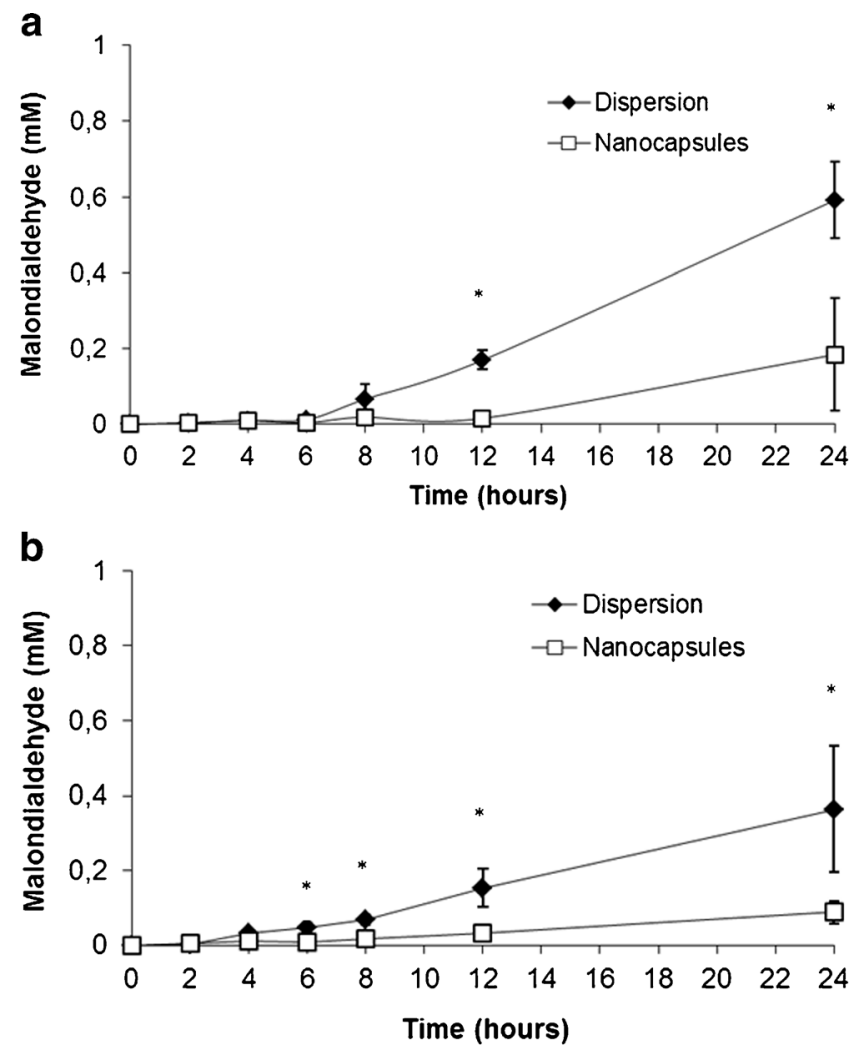

Fig. 4. Malondialdehyde formed as a function of time under UVA (a) and UVC (b) light. *Statistical difference between free oil dispersion and nanocapsules $(p<0.05)$ difference between formulations when aliquots were withdrawn after 12 and $24 \mathrm{~h}$ under light. For the UVC light, there is a significant difference between formulations when aliquots were withdrawn after $6,8,12$, and $24 \mathrm{~h}$ under light. In both conditions, the nanoencapsulated formulation presented less amount of malondialdehyde in the time intervals mentioned. So, the nanoencapsulation was able to protect the oil from oxidation, leading to a formulation of high stability. The property of the nanocapsules in reducing the degradation of encapsulated drug under UV light was previously shown $(13,15)$. However, no data about the oxidation of vegetable oils and the influence of the nanocapsules on this parameter were described so far. Such increase in the stability of vegetable oils is of extreme importance since their oxidation might lead to loss in activity besides formation of unpleasant odor.

\section{Development of Final Formulation for Cutaneous Use of Rose-Hip-Oil-Loaded Nanocapsules}

Final dermal formulations based on chitosan and containing rose-hip-oil-loaded nanocapsules were successfully obtained as shown in Fig. 5. Chitosan was chosen as hydrogelforming and film-forming polymer due to its wound healing and adhesion properties $(23,24,32)$. Chitosan gel containing polymeric nanocapsules was already described by our research group, presenting great skin adhesion (8) and nonirritant properties (33). Moreover, the adjuvants sodium pyrrolidone carboxylic acid (Na-PCA) and volatile silicone were added to the gel aiming to improve the sensorial properties of the formulation, as previously described (28). However, as far as we know, this is the first time that chitosan films with polymeric nanocapsules are produced.

The chitosan hydrogel (Fig. 5a) presented opaque aspect, due to the presence of nanocapsules, adequate homogeneity, and apparent good spreadability on the skin. The chitosan film

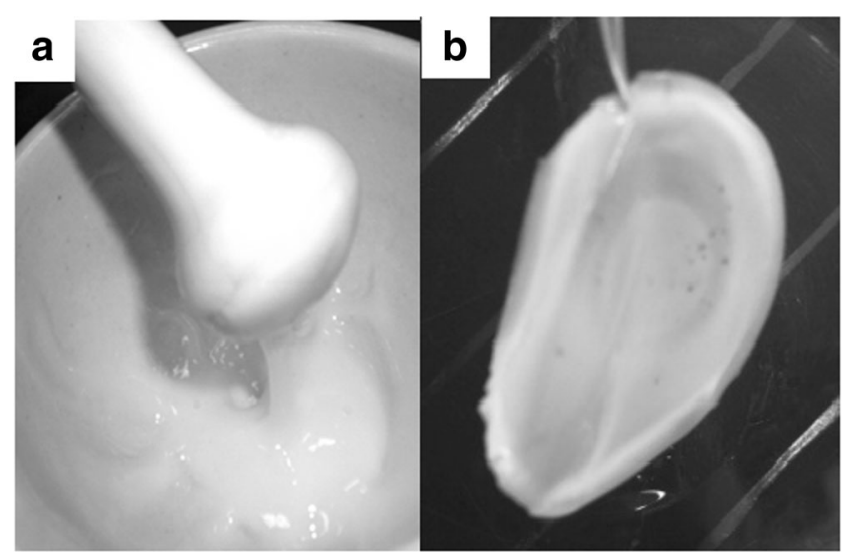

Fig. 5. Chitosan gel (a) and film (b) containing rose-hip-oil-loaded nanocapsules 


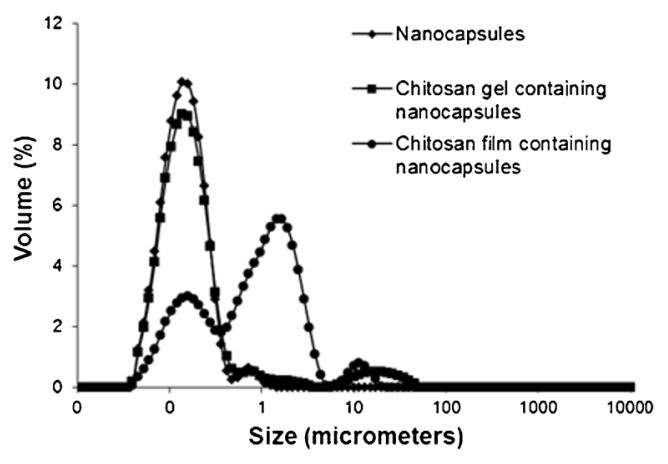

Fig. 6. Size distribution of nanocapsules incorporated into chitosan gel, into chitosan film, and right after production in colloidal dispersion

(Fig. 5b) presented also opaque aspect, besides apparent good mechanical resistance and good adhesion on the skin, the latter especially when the film was wet. It was previously described that the inclusion of polymeric nanoparticles to chitosan films increased their "time to break" and tensile strength (21). The films prepared were of $3 \mathrm{~cm}$ of diameter and presented initial weights of $209 \pm 8 \mathrm{mg}$ and thickness of $2.78 \pm 0.05 \mathrm{~mm}$. The chitosan gel and film containing nanocapsules formulation presented slightly acid $\mathrm{pH}$ ( $\mathrm{gel}=4.4 \pm 0.1$; film $=6.1 \pm 0.0)$, due to addition of lactic acid, necessary for the chitosan chain entanglement. The values were considered suitable for cutaneous use, due to acid properties of the stratum corneum (34).

Regarding the presence of nanocapsules in the chitosan dermal formulations, size distribution graphs were obtained by laser diffraction (Fig. 6). Analyzing the size distribution, it is possible to see that the gel and the film presented particles in the nanometric range, with similar size to that observed for the improved nanocapsules in aqueous colloidal dispersion. The results indicate that the incorporation of nanocapsules in chitosan formulations was successfully performed, and no damage is caused to the particles due to incorporation in the hydrogel, as previously described (8) or in the films. Although both of the chitosan formulations presented a population in the nanometric range, the particle size distribution of the chitosan gel and chitosan film containing nanocapsules presented distinct profiles. The chitosan film presented an additional population of close to $1 \mu \mathrm{m}$ of average diameter. Such larger particles can be agglomerated nanocapsules within the physically cross-linked chitosan. The chitosan chains are entangled in the gel, while they are physically cross-linked by tripolyphosfate in the film formulation. Such cross-linked chitosan presents a very tangled network (35), which can hinder the dispersion of nanocapsules into their primary size in water during the measurement of particle size distribution. The chosen vehicles based on chitosan appear to be suitable alternatives for carrying the rose-hip-oil-loaded polymeric nanocapsules.

The chitosan gel was additionally evaluated according to its rheological profile and its physical stability. The rheological data (shear stress vs. shear rate) were well fitted to the Ostwald flow model $(r>0.99)$, which corresponds to the pseudoplastic flow favoring the cutaneous applications of products (36). The consistence index was $31.75 \pm 1.73$ and the flow index was $0.72 \pm 0.01$. The average consistence value observed is higher than the values previously observed for chitosan gels containing polymeric nanocapsules and the adjuvants (PCA-Na and volatile silicone) (28), which were around 20. This difference is probably due the fact that the number of particles in the original aqueous suspension and posterior in the hydrogel is higher, when compared to the previous investigation. Regarding the physical stability of the formulation, Fig. 7 shows the delta backscattering obtained as mentioned in the "Nanoparticle Properties" section. No specific instability phenomena are detected for the chitosan hydrogel formulation, since the variation in the delta backscattering is under $10 \%$ (37). The incorporation of the rose-hip-oilloaded nanocapsules in a chitosan hydrogel reduced the creaming of the particles observed when these were in aqueous suspension, due to increase in viscosity of the system. Although the creaming did not interfere in the nanocapsules properties, its reduction was considered positive, since the creaming can interfere in the homogeneity of doses.

The chitosan film containing rose-hip-oil-loaded polymeric nanocapsules was additionally evaluated according to water absorption capacity. It was observed that the films decreased their weights in $23 \pm 2 \%$ after $30 \mathrm{~min}$ and $31 \pm 4 \%$ after $1 \mathrm{~h}$ in water immersion. After $3 \mathrm{~h}$ at the same condition, the weight decrease was kept at $32 \pm 2 \%$. The weight decrease observed in the first hour of immersion was probably due to residual substances, such as tripolyphosphate, being released from the films to the water, decreasing the weights of the films. After $3 \mathrm{~h}$, it was observed that the films kept their weights,

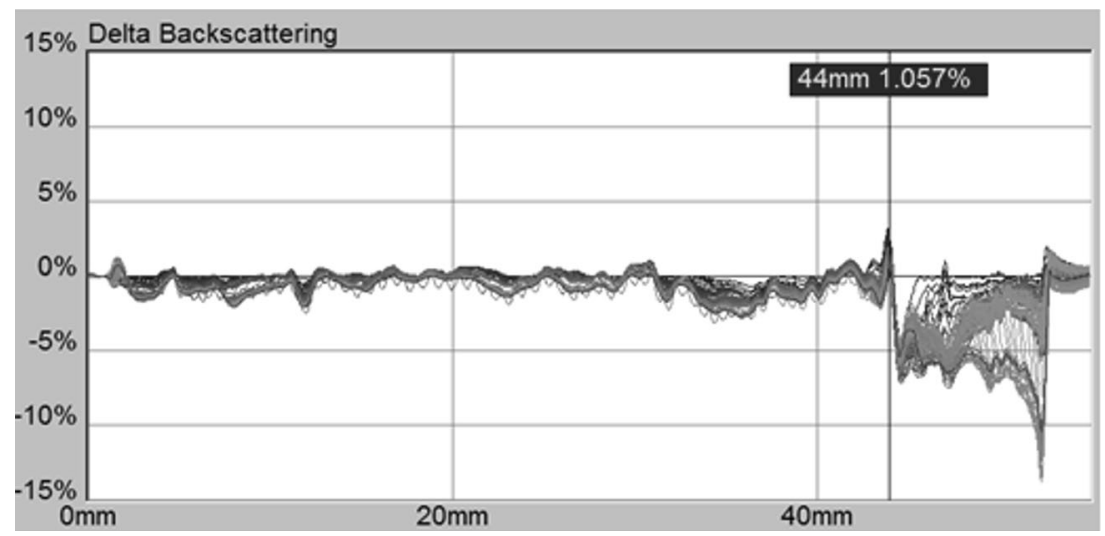

Fig. 7. Delta backscattering analysis of chitosan gel containing rose-hip-oil-loaded nanocapsules 
indicating stability of the films in water. No water absorption was observed, oppositely as described for previously reported chitosan films $(17,32,22)$. In the present investigation, the network formed by cross-linked chitosan and nanocapsules might have influenced the water absorption capacity of chitosan. Considering that the formulations are expected to be applied at an injured skin area, the absence of water absorption can be positive. Formulations that completely absorb the wound fluid, preventing a certain moisture level, may hinder the wound healing process (32).

\section{CONCLUSION}

The rose-hip-oil-loaded nanocapsules were improved by increasing the acetone and decreasing the rose-hip oil content during particle formation. It was possible to nanoencapsulate an effective dose of rose-hip oil and to protect the oil from UV oxidation. The nanoencapsulation of rose-hip oil allows its incorporation into aqueous formulations. The innovative final formulations of chitosan gel and chitosan film containing rosehip-oil-loaded nanocapsules presented suitable properties for the application on the skin, combining the advantages of the nanocapsules to the advantages of chitosan.

\section{ACKNOWLEDGMENTS}

RVC thanks CNPq/Brazil for her fellowship.

Conflict of interest The authors declare no conflict of interests.

\section{REFERENCES}

1. Franco D, Pinelo M, Sineiro J, Nuñez MJ. Processing of Rosa rubiginosa: extraction of oil and antioxidant substances. Bioresour Technol. 2007;98:3506-12. doi:10.1016/ j.biortech.2006.11.012.

2. Pareja B, Kehl H. Contribuición a la identificación de los principios activos en el aceite de Rosa aff. Rubiginosa L An Real Acad Farm. 1990;56:283-94.

3. Eurides D, Da Silva LAF, Daleck CR, Freitas PMC, Alves LB. Efecto del extracto de óleo de rosa mosqueda (Rosa aff. Rubiginosa) en la cicatrización de heridas cutáneas. Rev Electrónica Vet. 2011;12:1695-7504.

4. Dweck AC. Formulating with natural ingredients. Cosmetics Toiletries. 2001;116:57-60.

5. Guterres SS, Alves MP, Pohlmann AR. Polymeric nanoparticles, nanospheres and nanocapsules for cutaneous applications. Drug Target Insights. 2007;2:147-57.

6. Pardeike J, Hommoss A, Müller RH. Lipid nanoparticles (SLN, NLC) in cosmetic and pharmaceutical dermal products. Int $\mathbf{J}$ Pharm. 2009;366:170-84. doi:10.1016/j.ijpharm.2008.10.003.

7. Prow TW, Grice JE, Lin LL, Faye R, Butler M, Becker W, et al. Nanoparticles and microparticles for skin drug delivery. Adv Drug Deliv Rev. 2011;63:470-91. doi:10.1016/j.addr.2011.01.012.

8. Contri RV, Katzer T, Ourique A, Da Silva ALM, Beck RCR, Pohlmann AR, et al. Combined effect of polymeric nanocapsules and chitosan hydrogel on the increase of capsaicinoids adhesion to the skin surface. J Biomed Nanotechnol. 2014;10:820-30. doi:10.1166/jbn.2014.1752.

9. Jiménez MM, Pelletier J, Bobin MF, Martini MC. Influence of encapsulation on the in vitro percutaneous absorption of octyl methoxycinnamate. Int J Pharm. 2004;272:45-55. doi:10.1016/ j.ijpharm.2003.11.029.
10. Lboutounne H, Chaulet JF, Ploton C, Falson F, Pirot F. Sustained ex vivo skin antiseptic activity of chlorhexidine in poly(ecaprolactone) nanocapsule encapsulated form and as a digluconate. J Control Release. 2002;82:319-34.

11. Jeong I, Kim BS, Lee H, Lee KM, Kang SK, Yin CS, et al. Prolonged analgesic effect of PLGA-encapsulated bee venom on formalin-induced pain in rats. Int J Pharm. 2009;380:62-6. doi:10.1016/j.jpharm.2009.06.034.

12. Fontana MC, Rezer JFP, Coradini K, Leal DBR, Beck RCR. Improved efficacy in the treatment of contact dermatitis in rats by a dermatological nanomedicine containing clobetasol propionate. Eur J Pharm Biopharm. 2011;79:241-9. doi:10.1016/j.ejpb.2011.05.002.

13. Ourique AF, Pohlmann AR, Guterres SS, Beck RCR. Tretinoinloaded nanocapsules: preparation, physicochemical characterization, and photostability study. Int J Pharm. 2008;352:1-4. doi:10.1016/j.ijpharm.2007.12.035.

14. Külkamp IC, Paese K, Pohlmann AR, Guterres SS. Estabilização do ácido lipoico via encapsulação em nanocápsulas poliméricas planejadas para aplicação cutânea. Quim Nova. 2009;8:2078-84. doi:10.1590/S0100-40422009000800018.

15. Mazzarino L, Dora CL, Bellettini IC, Minatti E, Cardoso SG, Lemos-Senna E. Curcumin-loaded polymeric and lipid nanocapsules: preparation, characterization and chemical stability evaluation. Lat Am J Pharm. 2010;29:933-40.

16. Contri RV, Correa KLF, Fiel LA, Pohlmann AR, Guterres SS. Vegetable oils as core of cationic polymeric nanocapsules: influence on the physicochemical properties. J Exp Nanosci. 2013;8:8913-24. doi:10.1080/17458080.2011.620019.

17. Şenel S, Ikinci G, Kaş S, Yousefi-rad A, Sargon MF, Hincal AA. Chitosan films and hydrogels of chlorhexidine gluconate for oral mucosal delivery. Int J Pharm. 2000;193:197-203. doi:10.1016/ S0378-5173(99)00334-8.

18. Ruel-gariépy E, Leclairb G, Hildgenb P, Guptac A, Leroux JC, Thermosensitive chitosan-based hydrogel containing liposomes for the delivery of hydrophilic molecules. J Control Release. 2002;82:373-83. doi:10.1016/S0168-3659(02)00146-3.

19. Lakshimi PK, Devi GS, Bhaskaran S, Sacchidanand S. Niosomal mathotrexate gel in the treatment of localized psoriasis: phase I and phase II studies. Ind J Dermatol Venereol Leprol. 2007:3:157-61. doi:10.4103/0378-6323.32709.

20. Souto EB, Wissing SA, Barbosa CM, Müller RH. Evaluation of the physical stability of SLN and NLC before and after incorporation in hydrogel formulations. Eur J Pharm Biopharm. 2004;58:83-90. doi:10.1016/j.ejpb.2004.02.015.

21. Giovino C, Ayensu I, Tetteh J, Boateng JS. Development and characterisation of chitosan films impregnated with insulin loaded PEG- $b$-PLA nanoparticles (NPs): a potential approach for buccal delivery of macromolecules. Int J Pharm. 2012;428:143-51. doi:10.1016/j.ijpharm.2012.02.035.

22. Vimala K, Mohan YM, Sivudu KS, Varaprasad K, Ravindra S, Reddy NN, et al. Fabrication of porous chitosan films impregnated with silver nanoparticles: a facile approach for superior antibacterial application. Colloids Surf B: Biointerfaces. 2010;76:24858. doi:10.1016/j.colsurfb.2009.10.044.

23. Ueno H, Yamada H, Tanaka I, Kaba N, Matsuura M, Okumura $\mathrm{M}$, et al. Accelerating effects of chitosan for healing at early phase of experimental open wound in dogs. Biomaterials. 1999;20:140714. doi:10.1016/S0142-9612(99)00046-0.

24. Ueno H, Mori T, Fujinaga T. Topical formulations and wound healing applications of chitosan. Adv Drug Deliv Rev. 2001;52:105-15. doi:10.1016/S0169-409X(01)00189-2.

25. Rinaudo M. Chitin and chitosan: properties and applications. Prog Polym Sci. 2006;31:603-32. doi:10.1016/j.progpolymsci.2006.06.001.

26. Jäger E, Venturini CG, Poletto FS, Colomé LM, Pohlmann JPU, Bernardi A, et al. Sustained release from lipid-core nanocapsules by varying the core viscosity and the particle surface area. J Biomed Nanotechnol. 2009;5:130-40. doi:10.1166/jbn.2009.1004.

27. Külkamp IC, Rabelo BD, Berlitz SJ, Isoppo M, Bianchin MD, Schaffazick SR, et al. Nanoencapsulation improves the in vitro antioxidant activity of lipoic acid. J Biomed Nanotechnol. 2011;7:598-607. doi:10.1166/jbn.2011.1318.

28. Contri RV, Külkamp-Guerreiro IC, Krieser K, Pohlmann AR, Guterres SS. Applying the sensorial analyses in the development of a novel chitosan hydrogel containing polymeric nanocapsules for cutaneous use. J Cosmet Sci. 2014;65:1-16. 
29. Frank LA, Sandri G, D'Autilia F, Contri RV, Bonferoni MC, Caramella $\mathrm{C}$, et al. Chitosan gel containing polymeric nanocapsules: a new formulation for vaginal drug delivery. Int $\mathrm{J}$ Nanomedicine. 2014;9:3151-61. doi:10.2147/IJN.S62599.

30. Schaffazick SR, Pohlmann AR, Guterres SS. Nanocapsules, nanoemulsion and nanodispersion containing melatonin: preparation, characterization and stability evaluation. Pharmazie. 2007;62:354-60. doi:10.1691/ph.2007.5.6087.

31. Contri RV, Kaiser M, Poletto FS, Pohlmann AR, Guterres SS Simultaneous control of capsaicinoids release from polymeric nanocapsules. J Nanosci Nanotechnol. 2011;11:2398-406. doi:10.1166/jnn.2011.3521.

32. Sezer AD, Hatipoglu F, Cevher E, Ogurtan Z, Bas AL Akbuga $\mathrm{J}$,. Chitosan film containing fucoidan as a wound dressing for dermal burn healing: preparation and in vitro/in vivo evaluation. AAPS Pharm Sci Tech. 2007;E1-E8.

33. Contri RV, Frank LA, Kaiser M, Pohlmann AR, Guterres SS. Decreased irritation of capsaicinoids to human skin by means of nanoencapsulation. Int J Nanomedicine. 2014;9:951-62. doi:10.2147/IJN.S56579.

34. Schmid-wendtner MH, Korting HC. The $\mathrm{pH}$ of the skin surface and its impact on the barrier function. Skin Pharmacol Physiol. 2006;19:296-302.

35. Berger J, Reist M, Mayer JM, Felt O, Peppas NA, Gurny R. Structure and interactions in covalently and ionically crosslinked chitosan hydrogels for biomedical applications. Eur J Pharm Biopharm. 2004;57:19-34. doi:10.1016/S0939-6411(03)00161-9.

36. Guaratini T, Gianeti MD, Campos PMBGM. Stability of cosmetic formulations containing ésteres of vitamins $E$ and $A$ chemical and physical aspects. Int J Pharm. 2006;327:12-6. doi:10.1016/ j.ijpharm.2006.07.015.

37. Celia C, Trapasso E, Cosco D, Paolino D, Fresta M. Turbiscan Lab ${ }^{\circledR}$ Expert analysis of the stability of ethosomes ${ }^{\circledR}$ and ultradeformable liposomes containing a bilayer fluidizing agent. Colloids Surf B: Biointerfaces. 2009;72:155-60. doi:10.1016/ j.colsurfb.2009.03.007. 